\title{
Lettre à la rédaction : Fiche de renseignements cliniques en toxicologie d'urgence - Recommandations
}

\section{Recommendations for clinical information for toxicology laboratories}

Michel LAVIT ${ }^{(1)}$, Denis LAMIABLE ${ }^{(2)}$, Bernard CAPOLAGHI ${ }^{(3)}$

(1) Laboratoire de Pharmacocinétique et de Toxicologie Clinique, Hôpital de Rangueil, 1 , avenue Jean-Poulhès - 31403 TOULOUSE Cedex 4 (2) Laboratoire de Pharmacologie-Toxicologie, C.H.U. Maison Blanche, 45, rue Cognacq-Jay - 51092 REIMS Cedex (3) Laboratoire de Biologie, Hôpital Bel-Air, C.H.R. Metz-Thionville, rue du Friscaty - 57100 THIONVILLE

(Reçu le 5 novembre 2002 ; accepté le 23 novembre 2002)

Durant ces dernières années, les intoxications aiguës sont devenues un véritable problème de santé publique dans les pays industrialisés en raison de leur fréquence et de la multiplicité des produits responsables. Avec une incidence annuelle de $4 \%$ habitants (1), les intoxications médicamenteuses représentent à elles seules 50 à $60 \%$ de la totalité de ces intoxications et constituent une des premières causes d'urgence hospitalière et d'hospitalisation en réanimation médicale. Volontiers polymédicamenteuses, elles sont caractérisées par une forte prévalence des psychotropes, des antalgiques et des cardiotropes. Les intoxications non médicamenteuses, moins fréquentes mais plus graves, recouvrent des réalités très différentes : produits domestiques, agricoles, industriels...

Après exclusion d'autres causes, le diagnostic toxicologique repose avant tout sur une approche clinique regroupant l'anamnèse, l'examen clinique avec, si possible, individualisation de syndromes toxiques (anti- cholinergique, effet stabilisant de membrane, sérotoninergique...) témoin de l'évolution de la pharmacopée et des habitudes de prescription. Cette démarche est complétée par la mise en évidence de perturbations biologiques, critères indirects d'une intoxication aiguë, notamment pour les toxiques dits lésionnels. De plus, les investigations toxicologiques jouent un rôle important en permettant la confirmation ou non d'une hypothèse toxique, l'évaluation de la sévérité ou du pronostic d'une intoxication et la surveillance d'un traitement adapté (2).

Pour être réellement efficaces c'est à dire avoir un impact clinique reconnu, les analyses toxicologiques doivent obligatoirement découler d'une collaboration étroite entre le clinicien confronté à des présentations rarement pathognomoniques et le biologiste disposant d'un arsenal analytique de plus en plus performant. En d'autres termes, il apparaît fondamental de rationaliser les recherches toxicologiques afin d'éviter les opéra- 
tions systématiques et aveugles induisant une sécurité trompeuse et coûteuse (3). Ce concours multidisciplinaire, idéalement basé sur un échange d'informations en amont et en aval du processus analytique, assure une rentabilité optimale, compatible avec l'urgence toxicologique. Un des volets de cette collaboration clinicobiologique (4) (5) réside en la transmission par le clinicien d'un formulaire de demande joint systématiquement aux échantillons à analyser. Ce document a pour vocation d'aider l'analyste dans sa démarche. Cet aspect est, par ailleurs, réglementé par le Guide de Bonne Exécution des Analyses de Biologie Médicale (G.B.E.A.) qui spécifie que "les échantillons doivent ... être associés à une fiche de suivi médical comportant tous les renseignements nécessaires à la bonne exécution des analyses et à l'interprétation des résultats" (6).

Dans ce cadre, la Commission Toxicologie Hospitalière de la SFTA a mené une réflexion sur la constitution de ces fiches de renseignements cliniques afin de proposer une série de recommandations consensuelles, portant notamment sur les informations considérées comme indispensables pour toute investigation toxicologique.

La méthodologie a consisté, tout d'abord, en l'examen de quelques formulaires représentatifs de l'existant dans les différents centres. Tous comportent deux parties bien distinctes. La première, plus ou moins développée, est destinée aux données cliniques ; la seconde aux examens prescrits. La différence essentielle concerne la manière de remplir ces fiches. Certaines proposent aux prescripteurs de simples items à cocher, d'autres doivent être renseignées en texte libre. Dans tous les cas, elles reflètent le mode de fonctionnement spécifique de chaque laboratoire.

Après ces considérations sur la forme, la Commission s'est attachée à étudier la pertinence des différents renseignements pour ne retenir que ceux jugés incontournables et complémentaires à tout prélèvement biologique. Ces derniers peuvent alors être classés en quatre catégories :

\section{- Échantillon :}

* Nature : sang, urine, autre

* Date et heure du prélèvement

\section{- Contexte :}

* Type de l'intoxication : aiguë, surdosage

* Date et heure suspectées de l'intoxication

* Toxicomanie connue

* Médicaments et/ou toxiques en cause et quantité

* Traitement médicamenteux habituel

* Thérapeutique mise en œuvre

\section{- État clinique :}

* Neurologique : coma, convulsions, agitation, score de Glasgow, myosis - mydriase

* Cardiaque

* Respiratoire

\section{- Anomalies biologiques éventuelles}

Ainsi, cette fiche fournit au biologiste une aide précieuse dans la définition des priorités, pour le choix d'une approche analytique adaptée au patient dans un contexte d'urgence hospitalière. Toutefois, elle ne dispense pas d'une communication directe entre les différents partenaires, véritable pilier de la collaboration clinico-biologique.

Remerciements à tous les membres de la commission hospitalière de la Société Française de Toxicologie Analytique ayant collaboré à ce travail.

\section{Références}

1. Lambert H, Manel J, Bellou A, El Kouch S. Morbidité et mortalité par intoxications médicamenteuses aiguës en France. Rev. Prat. 1997 ; 47 : 716-20.

2. Lheureux P, Askenasi R, Maes V. Du bon usage du laboratoire de toxicologie 2ème partie: Utilité clinique et interprétation des résultats. Réan. Urg. $1996 ; 5(3)$ : 341 52.

3. Lheureux P, Maes V, Askenasi:R. Du bon usage du laboratoire de toxicologie 1ère partie : Aspects techniques. Réan. Urg. 1996 ; 5(2) : 87-95.

4. Capolaghi B, Moulsma M, Houdret N, Baud F. Stratégies analytiques en toxicologie d'urgence. Ann. Toxicol. Anal. 2000 ; 12(4) : 274-81.

5. Vincent F, Danel V. Quelle collaboration clinico-biologique pour la prise en charge des intoxications aiguës? Ann. Toxicol. Anal. $2000 ; 12(4)$ : 267-73.

6. Arrêté du 26 novembre 1999 relatif à la bonne exécution des analyses de biologie médicale. Journal Officiel, $\mathrm{N}^{\circ} 287$ du 11 décembre 1999, page 18441. 